\title{
Single-Iteration Algorithm for Compressive Sensing Reconstruction
}

\author{
Srdjan Stanković, Senior Member, IEEE, Irena Orović, Member, IEEE, \\ Ljubiša Stanković, Fellow, IEEE, and Andjela Draganić, Student Member, IEEE
}

\begin{abstract}
A single-iteration algorithm is proposed for the reconstruction of sparse signal from its incomplete set of observations. Recently, the reconstruction algorithms have been intensively developed within the Compressive Sensing framework. Most of the existing solutions are based either on $l_{1}$-norm optimization methods or greedy iterative procedures with a priori known number of components or predefined number of iterations. We propose a simple non-iterative algorithm based on the analysis of noise-effect that appears in the frequency domain as a consequence of missing samples. The noise variance can be related and controlled by the number of missing samples. Accordingly, it is possible to keep the level of spectral noise below the signal components, such as to be able to accurately detect signal support and to reconstruct the entire signal. The theory is proven on various examples with multicomponent signals.
\end{abstract}

Keywords - Compressive Sensing, missing samples, DFT, reconstruction algorithms.

\section{INTRODUCTION}

$\mathrm{N}$ OWADAYS, one of the most challenging topics and a key issue in various applications such as data compression, source separation, noise reduction, and more recently Compressive Sensing (CS) is finding a sparse linear decomposition of a given signal [1]-[3]. Particularly, in the case of $\mathrm{CS}$, the idea is to reconstruct the entire signal from its small set of random available samples by searching for the best sparse approximation. Various CS signal reconstruction methods have been proposed in the literature [4]-[9], and among them we may define two general approaches. The first one is $l_{1}$ norm based minimization approach which is solved using convex programming [4], [5]. On the other hand, there are much faster approaches, so called greedy algorithms, which are iterative procedures that decrease the approximation error by relaxing the sparsity constraint. The most commonly used among them is the Orthogonal Matching Pursuit

Paper received March 19, 2014; accepted April 4, 2014. Date of publication July 31,2014 . The associate editor coordinating the review of this manuscript and approving it for publication was Prof. Branimir Reljin.

This paper is a revised and expanded version of the paper presented at the 21th Telecommunications Forum TELFOR 2013.

This work has been supported by the project CS-ICT (New ICT Compressive sensing based trends applied to: multimedia, biomedicine and communications), funded by Montenegrin Ministry of Science.

The authors are with the Faculty of Electrical Engineering, University of Montenegro, Džordža Vašingtona bb. 18000 Podgorica, Montenegro. The corresponding author: Irena Orović (tel: +382 67516795 , e-mail: irenao@ac.me).
(OMP) [8], [9], together with a number of its variations such as Gradient Pursuit (GP), Compressive Sampling Matching Pursuit (CoSaMP), etc. Although they are faster and simpler than convex optimization algorithms, the greedy methods may usually assume that the number of signal components is a priori known. Otherwise, the stopping criterion for iterations can be based on mean square error [10]. The error value should be estimated empirically for each particular application, since it needs to be specified in advance. Through iterations, the greedy algorithms approximate the coefficients and the support of the original signal, until the stopping criterion is met.

In this paper, we propose a single-iteration algorithm based on the analysis of effects caused by missing samples. We start from the assumption that the considered compressive sampled signals are sparse in the Fourier transform domain. The side effects caused by having an incomplete set of time observations instead of a full data set are modeled as spectral noise characterized by a mean value and variance. As long as we are able to keep the noise effects below the signal components level in the Fourier domain, we can provide the entire signal reconstruction within a single iteration. The proposed solution also defines an optimal number of available measurements, which is required to recover a signal with a low probability of error. Here, it is important to emphasize that the proposed concept is not only applicable to the signal and its Fourier transform, but can be extended to the time-frequency representations being crucial in various real applications including radars, biomedicine, multimedia, [12]-[14], etc. Namely, most of the existing time-frequency representations are obtained as the Fourier transform of windowed auto-correlation function. In this case, the proposed concept could be applied to the windowed auto-correlation function segments.

The paper is organized as follows: The theoretical background about the Compressive Sensing is given in Section II. The single-iteration reconstruction algorithm is proposed in Section III. The experimental results are presented in Section IV, while the Concluding remarks are given in Section V.

\section{COMPRESSIVE SENSING}

Compressive sensing is usually applied when dealing with signals that need a high sampling rate, since the acquisition of these signals may require a high number of sensors, and large storage and transmission resources. In these circumstances, it is useful to explore the possibility of sampling at far lower rates, and, when required, to 
reconstruct the entire signal from the small set of measurements. For this purpose, we need a random sampling procedure that provides incoherent measurements. Also, the reconstruction should be done in the domain where the signal is sparse. Sparsity means that the signal can be represented by a small number of nonzero coefficients. The mathematical foundation of CS lies in the fact that it is possible to reconstruct a sparse signal from an underdetermined linear system of equations, and that this can be done in a computationally efficient manner via convex programming [5]. Consider a signal $\mathbf{s}$ that can be represented in a certain basis $\Psi=\{\Psi\}_{k=1}^{N}$, using the weighting coefficients $x_{k}$ :

$$
s=\sum_{k=1}^{N} x_{k} \Psi_{k} .
$$

The previous relation can be written also in the vector form as follows:

$$
\mathbf{s}=\boldsymbol{\Psi} \mathbf{x},
$$

where $\boldsymbol{\Psi}=\left[\Psi_{1}, \Psi_{2}, \ldots, \Psi_{N}\right]$ is a full rank $N \times N$ matrix. One example can be a finite-length discrete signal represented using discrete sinusoids in a limited bandwidth. The matrix $\boldsymbol{\Psi}$ would be actually the discrete Fourier transform (DFT) matrix. In CS, we are interested in any basis that allows a sparse representation of $\mathbf{s}$. Generally, it is assumed that $\mathbf{s}$ is not available. Instead of the whole signal $\mathbf{s}$, we are actually dealing with a small set of $M$ randomly sensed measurements, where $M<<N$. A set of random measurements is selected using the random measurement matrix $\Phi[1]-[3]$, as follows:

$$
\mathbf{y}=\boldsymbol{\Phi} \text {. }
$$

Accordingly, we may write:

$$
\mathbf{y}=\boldsymbol{\Phi} \Psi \mathbf{x}=\boldsymbol{\Theta x}
$$

Here, the matrix $\boldsymbol{\Theta}$ is called the CS matrix of size $M \times N$, $\mathbf{y}$ is an $M \times 1$ vector of available measurements, while $\mathbf{x}$ is a desired $N \times 1$ vector of transform domain coefficients. Consequently, in order to obtain the reconstructed signal, we need to solve the underdetermined system of $M$ linear equations with $N$ unknowns. It is obvious that this system may have infinitely many solutions, but the idea is to search for the sparsest one. For this purpose, various optimization algorithms based on $\ell_{0}$ - norm minimization were used. However, due to the problem complexity, the $\ell_{0}$ - norm minimization has been replaced in practical applications with the $\ell_{1}$ - norm minimization, leading to near-optimal solutions. The $\ell_{1}$-norm minimization problem in CS can be defined as follows [4]:

$$
\min \|\hat{\boldsymbol{x}}\|_{\ell_{1}} \text { subject to } \boldsymbol{y}=\Theta \hat{\boldsymbol{x}}
$$

The above minimization can be solved by using convex optimization algorithms such as Basis Pursuit algorithm [15],[16], with some of the commonly used solvers such as simplex and interior point methods (e.g., primal-dual interior point method). However, the complexity of this realization is still high. Therefore, for real-time processing, the greedy algorithms, such as OMP and CoSaMP, have been widely used in the applications. These algorithms represent iterative approximate solutions. For instance, the
OMP algorithm includes the best fitting component in each iteration [8]-[10],[17], until the stopping criterion is met. The stopping criterion is usually related to the known number of signal components, or alternatively, the predefined reconstruction error. The greedy algorithms are generally far less complex than convex optimizers, but the convergence to the optimal solution is not guaranteed.

\section{Single-Iteration Reconstruction Algorithm (SIRA)}

In this Section we propose a single-step (non-iterative) algorithm for the reconstruction of compressive sensed signals. The proposed algorithm is based on the assumption that the observed signals are sparse in the DFT domain. As a consequence of having an incomplete set of signal samples, the DFT domain representation of the compressive sampled signal is not sparse and it is affected by noise-effect. Namely, the missing samples in the time domain (observation domain) will produce a certain kind of noise in the spectral domain (DFT domain) which deteriorates signal representation [18],[19]. A larger number of missing samples produces larger noise variance, ruining the signal's sparsity. Consequently, signal components detection becomes more difficult.

In order to identify the signal support (the positions of signal components) which is necessary for efficient signal reconstruction, we need to model the induced noise-effect. Hence, to provide automatic components detection and reconstruction when dealing with a compressive sensed signal, we need to start from the expression which relates the number of missing samples to the spectral noise variance. Let us assume that the signal $\mathbf{s}=s(n), n=1, \ldots, N$ consists of $K$ frequency components defined by the amplitudes $A i$ and frequencies $k i, i=1, \ldots, K$. The DFT of this signal is defined as:

$$
S(k)=\sum_{n=0}^{N-1} \sum_{i=0}^{K} A_{i} e^{-2 \pi j\left(k-k_{i}\right) n / N} .
$$

From the previous relation, we can observe the set of samples $\mathbf{h}$ defined as:

$$
h(n)=\left\{\sum_{i=0}^{K} A_{i} e^{-2 \pi j\left(k-k_{i}\right) n / N}, n=0, \ldots, N-1\right\},
$$

where $\sum_{n=0}^{N-1} h(n)=0$ holds. Furthermore, consider a set $\mathbf{y}$ of $M$ available samples from $\mathbf{h}$ corresponding to the CS signal. The DFT over the available samples can be written as follows:

$$
F(k)=\sum_{n=1}^{M} y(n)=\sum_{n=1}^{M} h(n)-v(n) .
$$

Note that at the positions of missing samples, the noise can be modeled as $v(n)=h(n)$. The mean value of DFT vector $F$ can be written as:

$$
E\{F\}=\sum_{i=1}^{K} M A_{i} \delta\left(k-k_{i}\right),
$$

whereas the variance of the DFT values at the non-signal positions can be calculated according to [17]:

$$
\sigma^{2}=\operatorname{var}\left\{F_{k \neq k_{i}}\right\}=\frac{M(N-M)}{(N-1)} \sum_{k=1}^{K} A_{i}^{2} .
$$


The DFT values at the non-signal positions (noise-alone positions) are Rayleigh distributed:

$$
f(z)=\frac{2 z}{\sigma^{2}} e^{-z^{2} / \sigma^{2}}, z \geq 0 .
$$

Since the smallest DFT component is equal to $M A_{\min }$, then using the Rayleigh distribution, we can define the probability that $N-K$ DFT values corresponding to spectral noise (noise-alone DFT positions) are all below the lowest component $M A_{\text {min }}$ :

$$
P_{e}=1-\left(1-e^{-\frac{\left|X_{\min }\right|}{\sigma^{2}}}\right)^{N-K} \approx 1-\left(1-e^{-\frac{M^{2} A_{\min }^{2}}{\sigma^{2}}}\right)^{N} .
$$

The previous expression can be used as an approximate form of error probability $P_{e}$ (probability of wrong detection of signal component), which is a basis of signal reconstruction algorithm summarized below.

\section{The proposed algorithm for signal reconstruction - Single-iteration reconstruction}

1. Set desired value for the probability of error $P_{e}$. It is recommended to choose a high value for $P_{e}$, in order to provide successful signal reconstruction (e.g. $P_{e}=10^{-2}$ ).

2. For a given $M, N$ and the total signal energy $\sum_{i=1}^{K} A_{i}^{2}$, calculate the variance of the DFT values at the non-signal positions.

3. Having $P_{e}$ and $\sigma^{2}$, and assuming that the lowest signal component has the amplitude $A_{\text {min }}$, determine the optimal number of available samples using (10):

$$
M_{\text {opt }} \geq \arg \min \left\{P_{e}\right\} \text {. }
$$

4. Calculate the noise threshold Thresh according to:

$$
\text { Thresh }=\sqrt{-\sigma^{2} \log \left(1-\sqrt[N]{P_{e}}\right)} .
$$

5. Calculate the initial DFT vector $\mathbf{X}$ that corresponds to the set of $M$ available measurements.

6. Find vector $\mathbf{k}$ of positions of DFT components higher than threshold:

$$
\mathbf{k}=\arg \{|X|>\text { Thresh }\} \text {. }
$$

7. Calculate the exact DFT values at positions $\mathbf{k}$ by solving the system in the least square sense:

$$
\mathbf{X}=\left(\Theta^{*} \Theta\right)^{-1} \Theta^{*} \mathbf{y} \text {, }
$$

where $\mathbf{y}$ represents the vector of available samples from original signal, while CS matrix $\Theta$ is obtained from the DFT matrix using columns that correspond to the frequencies $\mathbf{k}$ and rows corresponding to $M$ available measurements.

\section{EXAMPLES}

\section{A. SIRA application on the signal Fourier transform}

Example 1: The aim of this example is to provide the experimental evaluation of the proposed analysis and algorithm. First, let us observe a signal consisting of three components, with different amplitudes, defined as:

$$
s(n)=A_{1} \mathrm{e}^{j 2 \pi n f_{1} / N}+A_{2} \mathrm{e}^{j 2 \pi n f_{2} / N}+A_{3} \mathrm{e}^{j 2 \pi n f_{3} / N},
$$

where: $A_{1}=0.3, A_{2}=0.1, A_{3}=0.6, f_{1}=32, f_{2}=128, f_{3}=256$, and $n=1: N$. The total number of samples is 512 . Based on (10), the optimal number of samples for signal component detection can be determined. Here, the $P_{e}$ is set to 0.1 and $M_{\text {opt }}$ is calculated according to $M_{\text {op }} \geq \operatorname{argmin}\left\{P_{e}\right\}$, for each signal component. The optimal number of samples required for detection of each considered signal component is illustrated in Fig. 1. A higher amplitude requires a smaller number of samples, and vice versa. As it can be seen from Fig. 1, the third component with the highest amplitude $A_{3}=0.6$, requires the smallest number of samples, i.e., $M=15$, while components with amplitudes $A_{l}=0.3$ and $A_{2}=0.1$ require $M=65$ and $M=296$ samples, respectively. The initial and reconstructed FT when using a different number of available samples $M$ are shown in Fig. 2. In Fig. 2a, the component with the highest amplitude $A_{3}$ is detected and reconstructed, by using $M=15$. When we increase $M$ to 65 , the second component $\left(A_{1}\right)$ is detected-Fig. 2b. In Fig. 2c the third component is detected after we increased the number of measurements to $M=296$. Note that, in order to reconstruct the entire signal, the optimal number of samples $M_{\text {opt }}$ has to be calculated according to the amplitude of the smallest component. Therefore, $M_{\text {opt }}$ should be 296 in this case.

The threshold is marked by a horizontal line in Fig. 2. Having in mind $(8)$ and $\sqrt{-\sigma^{2} \log \left(1-\sqrt[N]{P_{e}}\right)}$, it can be concluded that the threshold will increase as $M$ increases, because the signal components will be more enhanced compared to noise.

Table 1 shows $M_{\text {opt }}$ and corresponding thresholds for the same signal and for different probabilities of error. As it is expected, the number of optimal samples per component decreases as the probability of error increases.

TABLE 1: THRESHOLD AND OPTIMAL NUMBER OF SAMPLES, FOR DIFFERENT AMPLITUDES AND PROBABILITIES OF ERROR.

\begin{tabular}{c|c|c|c}
\hline $\begin{array}{c}\text { Probability } \\
\text { of error }\end{array}$ & Amplitude & $\begin{array}{c}\text { Optimal } \\
\text { number } \\
\text { Mopt }\end{array}$ & Threshold \\
\hline \multirow{3}{*}{$P_{e}=0.001$} & 0.6 & 22 & 0.0126 \\
& 0.3 & 88 & 0.0235 \\
$P_{e}=0.01$ & 0.1 & 337 & 0.0295 \\
\hline & 0.6 & 18 & 0.0120 \\
$P_{e}=0.1$ & 0.3 & 78 & 0.0234 \\
& 0.1 & 318 & 0.0316 \\
\hline & 0.6 & 15 & 0.0118 \\
$P_{e}=0.9$ & 0.3 & 65 & 0.0232 \\
& 0.1 & 296 & 0.0344 \\
\hline
\end{tabular}




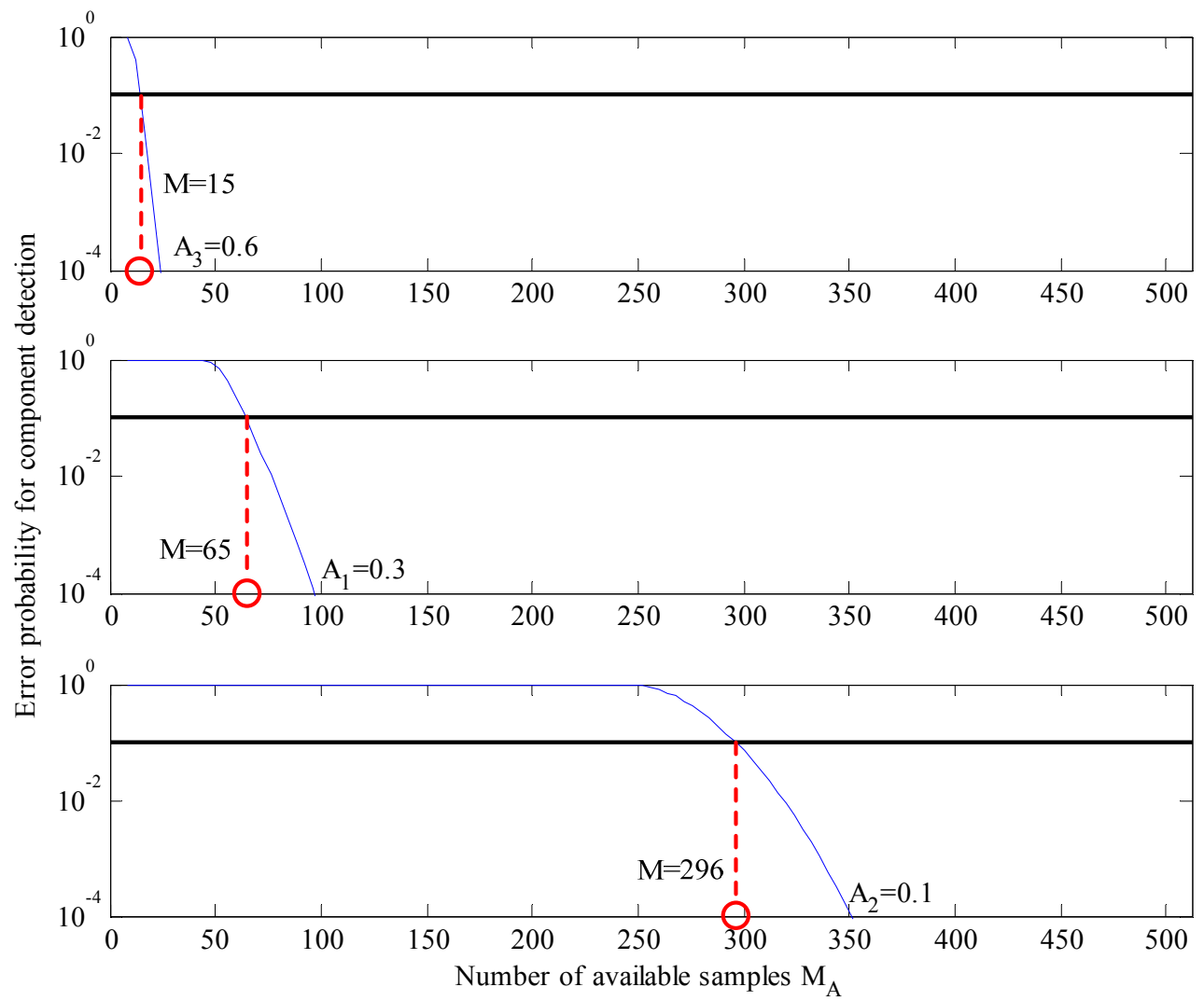

Fig. 1. Optimal number of available samples which assures $P_{e}=10^{-1}$, for different amplitudes of components.
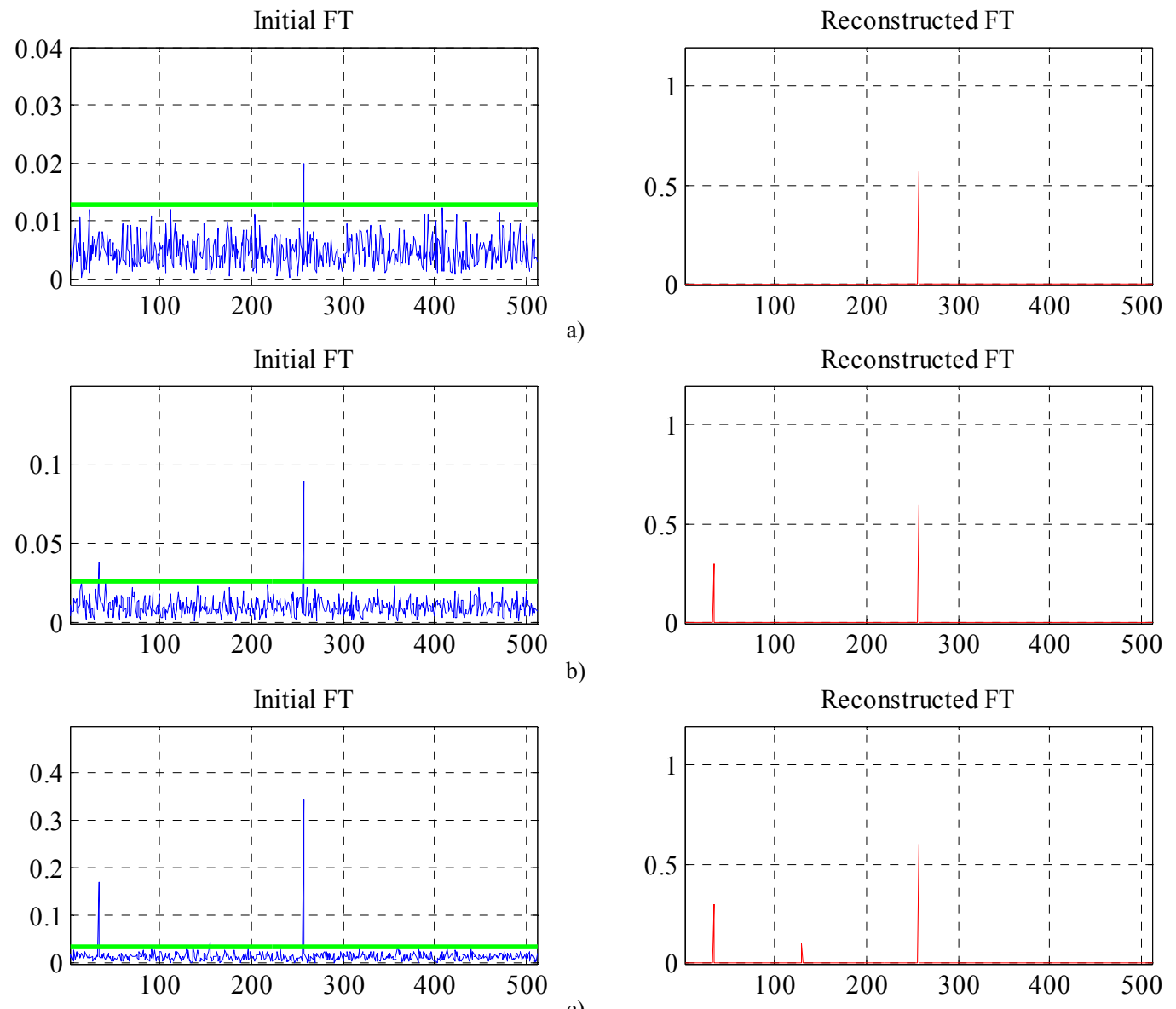

Fig. 2: Initial FT (left column) and reconstructed FT (right column) for different numbers of available samples: a) $M=15$, b) $M=65$, c) $M=296$. 
Example 2: Consider now the signal consisting of 10 components, defined as follows:

$$
s(n)=\sum_{i=1}^{10} A_{i} \mathrm{e}^{j 2 \pi n f_{i} / N} .
$$

The signal amplitudes are $\mathrm{A}_{1}=1.5, \mathrm{~A}_{2}=2.5, \mathrm{~A}_{3}=2, \mathrm{~A}_{4}=1.75$, $\mathrm{A}_{5}=2.25, \mathrm{~A}_{6}=3.5, \mathrm{~A}_{7}=2.15, \mathrm{~A}_{8}=3.25, \mathrm{~A}_{9}=3.75, \mathrm{~A}_{10}=3.4$, $\mathrm{A}_{11}=2.2, \mathrm{~A}_{12}=2.3, \mathrm{~A}_{13}=2.8, \mathrm{~A}_{14}=3.3$. Probability of error is set to $10^{-2}$ and the optimal number of samples should be determined based on the value of $P_{e}$. The strongest component could be detected with a small number of samples $M=30$, as it can be seen from Fig. 3 .

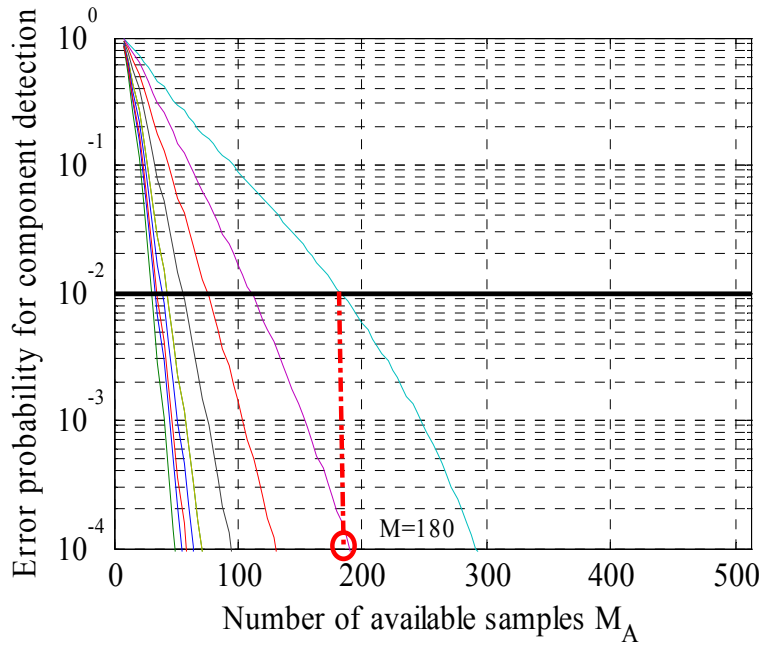

Fig. 3 Optimal number of available samples for $P_{e}=10^{-2}$ and different components amplitudes $(M=180$ for the smallest one).

The number of optimal samples required for successful reconstruction is chosen for a minimal amplitude component and it is equal to 180 for a chosen value of probability (Fig. 3). The initial and reconstructed FT are shown in Fig. 4. From the previous two examples it can be concluded that the reconstruction accuracy does not depend on the number of signal components, but only on the smallest component amplitude and chosen threshold (i.e. probability of error).

\section{A. SIRA application in the time-frequency domain}

Example 1: In this example we observe the application of the proposed reconstruction algorithm to the timefrequency representation. For the sake of simplicity we observe the short-time Fourier transform case (STFT) applied to the signal in the form:

$$
s(t)=3.1 e^{64 j \pi t}+3.2 e^{24 j \pi t}+3.15 e^{-96 j \pi t}+3 e^{128 j \pi t}
$$

The window width used in the STFT calculation is 128 samples. Signal is reconstructed by using a certain number of samples from each windowed signal part. The $25 \%$ of samples are considered as available within each windowed signal part. The spectrogram (SPEC) calculated from the available samples is shown in Fig. 5. Note that due to the missing samples, the SPEC has visible drawbacks reflected as noise in the time-frequency plane. Therefore, we need to apply the proposed reconstruction algorithm separately to each windowed signal part in order to reconstruct the entire representation. The results of reconstruction are shown in Fig. 6. Note that the components in the time-frequency plane are perfectly reconstructed.
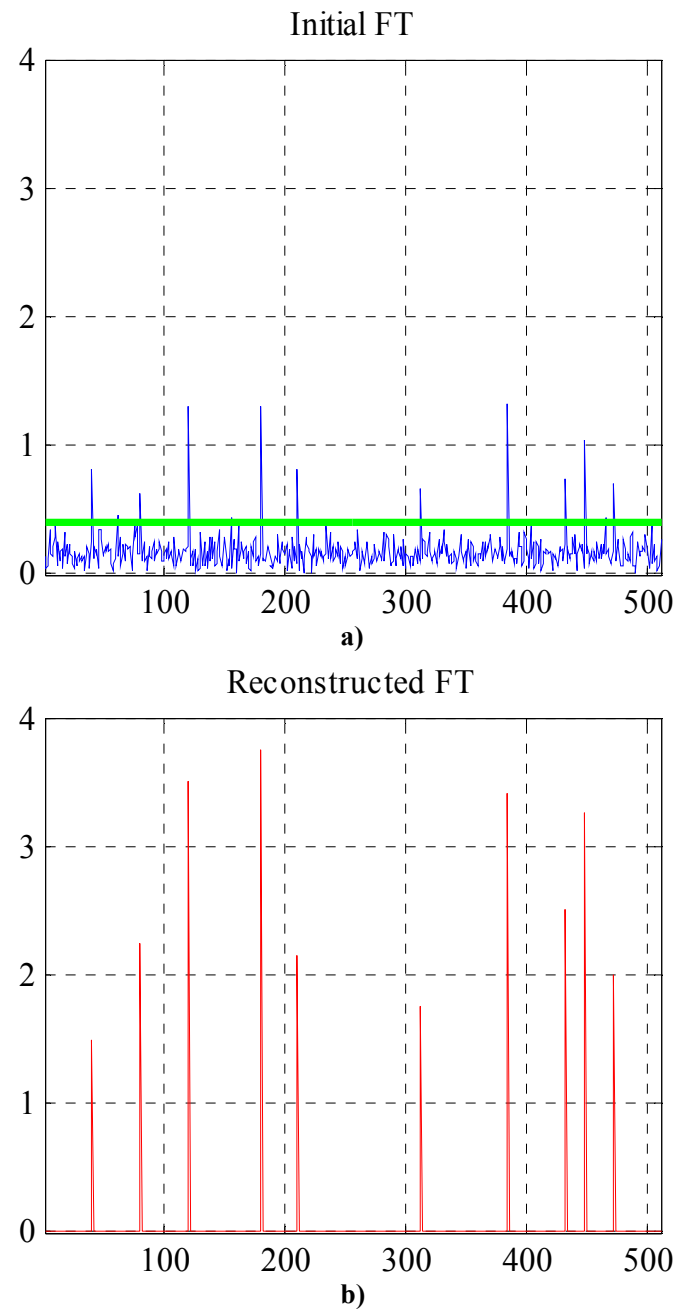

Fig. 4 a) Initial FT, b) Reconstructed FT by using $M=180$ samples and for $P_{e}=10^{-2}$.

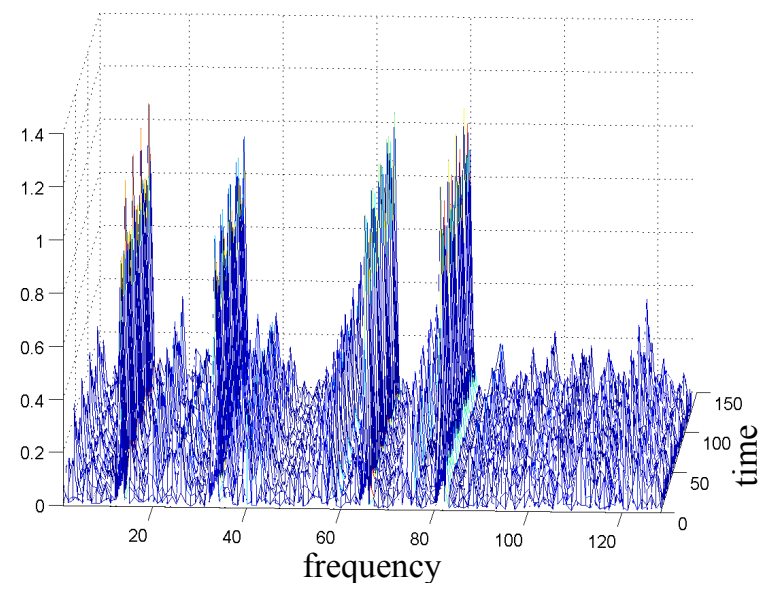

Fig. 5. SPEC calculated using only the available set of samples.

\section{CONCLUSION}

A single-iteration algorithm for Compressive Sensing reconstruction is proposed. Unlike most of the previously designed algorithms, the proposed solution is able to detect and reconstruct all signal components at once. For that purpose, we need to calculate the optimal number of 
available measurements that will assure total signal reconstruction with a desired low probability of error. The proposed method focuses on signals that are sparse in the DFT domain, but it has been shown that the proposed concept can be used even for time-frequency representations, where we might assume that the signal is sparse within the windowed segments.

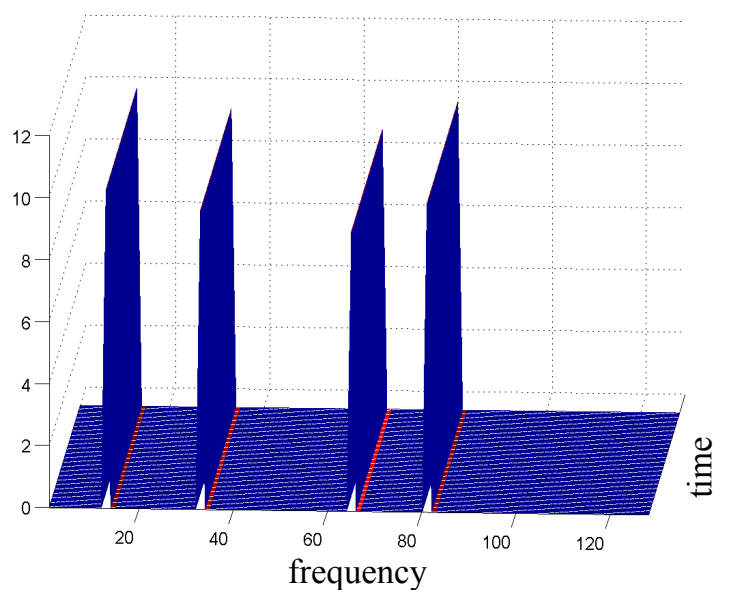

Fig. 6. The reconstructed SPEC by using the proposed algorithm.

\section{REFERENCES}

[1] D. Donoho, "Compressed sensing," IEEE Trans. on Information Theory, vol. 52, no.4, pp. 1289 - 1306, 2006.

[2] Baraniuk, R.: 'Compressive sensing' IEEE SP Magazine, 2007, 24, (4), pp. 118-121.

[3] S. Stankovic, I. Orovic, E. Sejdic, Multimedia Signals and Systems, Springer-Verlag, New York, 2012.

[4] E. Candes, and J. Romberg, L1-magic : Recovery of Sparse Signals via Convex Programming,

http://www.acm.caltech.edu/11magic $\wedge \#$ code, 2005, pp. 1-19

[5] S.P. Boyd, L. Vandenberghe, Convex optimization, Cambridge Univ. Press.
[6] S. Stankovic, LJ. Stankovic, I. Orovic, "A Relationship between the Robust Statistics Theory and Sparse Compressive Sensed Signals Reconstruction," IET Signal Processing, Special issue on Compressive sensing and robust transforms, May 2014.

[7] LJ. Stankovic, I. Orovic, S. Stankovic, M. Amin, "Compressive Sensing Based Separation of Non-Stationary and Stationary Signals Overlapping in Time-Frequency," IEEE Transactions on Signal Processing, vol. 61, no. 18, pp. 4562-4572, Sept. 2013.

[8] J. A. Tropp, A. C. Gilbert, "Signal Recovery From Random Measurements Via Orthogonal Matching Pursuit," IEEE Transaction on Information Theory, vol. 53, no.12, 2007.

[9] T. Zhang, "Sparse Recovery with Orthogonal Matching Pursuit Under RIP," IEEE Trans. on Inform. Theory, vol. 57, no.9, 2011

[10] M. Elad, Sparse and Redundant Representations: From Theory to Applications in Signal and Image Processing, Springer 2010.

[11] I. Orovic, M. Orlandic, S. Stankovic, Z. Uskokovic, "A Virtual Instrument for Time-Frequency Analysis of Signals with Highly Non-Stationary Instantaneous Frequency," IEEE Transactions on Instr. and Measurements, vol. 60, no. 3, pp. 791 - 803, 2011.

[12] B. Boashash, "Time'Frequency Signal Analysis" in S. Haykin, editor, Advances in Spectral Estimation and Array Processing, Prentice Hall, pp. 418-517, 1991.

[13] I. Orovic, S. Stankovic, "Improved Higher Order Robust Distributions based on Compressive Sensing Reconstruction," IET Signal Processing, 2014

[14] I. Orovic, S. Stankovic, T. Thayaparan, "Time-Frequency Based Instantaneous Frequency Estimation of Sparse Signals from an Incomplete Set of Samples," IET Signal Processing, Special issue on Compressive Sensing and Robust Transforms, 2014.

[15] S. S. Chen, D. L. Donoho, and M. A. Saunders, "Atomic decomposition by basis pursuit," SIAM Review, vol. 43, no. 1, pp. 129-159, 2001.

[16] D. L. Donoho and Y. Tsaig, "Fast solution of 11-norm minimization problems when the solution may be sparse," IEEE Transactions on Information Theory, vol. 54, no. 11, pp. 4789-4812, 2008.

[17] J. Tropp, "Greed is good: Algorithmic results for sparse approximation," IEEE Transactions on Information Theory, vol. 50, no. 10, pp. 2231-2242, 2004

[18] LJ. Stankovic, S. Stankovic, M. Amin, "Missing Samples Analysis in Signals for Applications to L-estimation and Compressive Sensing," Signal Processing, vol. 94, Jan 2014, pp. 401-408, 2013

[19] S. Stanković, I. Orović, and L. Stanković, "An Automated Signal Reconstruction Method based on Analysis of Compressive Sensed Signals in Noisy Environment", Signal Processing, accepted for publication. 